\title{
On the statistics of flame stretch in turbulent premixed jet flames in the thin reaction zone regime at varying Reynolds number
}

\author{
Stefano Luca ${ }^{\mathrm{a}, *}$, Antonio Attili ${ }^{\mathrm{b}}$, Ermanno Lo Schiavo ${ }^{\mathrm{c}, \mathrm{d}}$, Francesco Creta $^{\mathrm{d}}$, Fabrizio Bisetti ${ }^{\mathrm{e}, \mathrm{a}}$ \\ ${ }^{a}$ King Abdullah University of Science and Technology (KAUST), Clean Combustion Research Center (CCRC), Thuwal 23955, Saudi Arabia \\ ${ }^{b}$ Institute for Combustion Technology, RWTH Aachen University, Aachen, Germany \\ ${ }^{c}$ CERFACS, 42 Avenue G. Coriolis, 31057 Toulouse Cedex, France \\ ${ }^{d}$ Department of Mechanical and Aerospace Engineering, University of Rome "La Sapienza", Rome, Italy \\ ${ }^{e}$ Department of Aerospace Engineering and Engineering Mechanics, University of Texas at Austin, Austin, TX 78712, USA
}

\begin{abstract}
Direct Numerical Simulations (DNS) are conducted to study the statistics of flame surface stretch in turbulent jet premixed flames. Emphasis is placed on the rates of surface production and destruction and their scaling with the Reynolds number. Four lean methane/air turbulent slot jet flames are simulated at increasing Reynolds number and up to $\operatorname{Re} \approx 22 \times 10^{3}$, based on the bulk velocity, slot width, and the reactants' properties. The Karlovitz number is held approximately constant and the flames fall in the thin reaction zone regime. The simulations feature finite rate chemistry and mixture-average transport. Our data indicate that the area of the flame surface increases up to the streamwise position corresponding to $80 \%$ of the average flame length and decreases afterwards as surface destruction overtakes production. It is observed that the tangential rate of strain is responsible for the production of flame surface in the mean and surface destruction is due to the curvature term. In addition, it is found that these two terms are both significantly larger than their difference, i.e., the net surface stretch. The statistics of the tangential strain rate are in good agreement with those for infinitesimal material surfaces in homogeneous isotropic turbulence. Once scaled by the Kolmogorov time scale, the means of both contributions to stretch are largely independent of location and equal across flames with different values of the Reynolds number. Surface destruction is due mostly to propagation into the reactants where the surface is folded into a cylindrical shape with the center of curvature on the side of the reactants. The joint statistics of the displacement speed and curvature of the reactive surface are nuanced, with the most probable occurrence being that of a negative displacement speed of a flat surface, while the surface averaged displacement speed is positive as expected.
\end{abstract}

Keywords:

Turbulent premixed flames, Direct Numerical Simulation, Flame Surface Density, Flame Stretch

\footnotetext{
${ }^{*}$ Corresponding author

Email address: stefano. luca@kaust.edu.sa (Stefano Luca)
} 


\section{Introduction}

The characterization of the burning rate and spatial distribution of reactants and products is a primary objective in the analysis and design of technical combustion devices. It is generally understood that turbulence affects premixed combustion according to two mechanisms $[1,2]$. Turbulence disrupts the local balance between reaction and diffusion, altering the burning rate of the reactive front; in addition, turbulence wrinkles, stretches, and folds the flame surface, leading to an enhancement of the overall area of the flame.

In this work, we are concerned with the study of the growth rates of the area of the flame surface brought by turbulence, rather than with modifications to flame propagation. To this end, we conduct Direct Numerical Simulations (DNS) of turbulent premixed slot jet flames, where turbulence is driven by mean shear [3$6]$, in order to investigate flames in the thin reaction zone regime at moderate values of the Karlovitz number. While the Karlovitz number is kept approximately constant, the Reynolds number is varied by increasing the physical size of the jet. Our analysis, which is based on the surface density function (SDF) formalism [7], focuses on the spatial distribution of flame stretch and on the scaling of the growth rates of the flame area with Reynolds number.

The generation and destruction of surface is tied to velocity gradients and linked directly with the kinematics of turbulence [8]. It is well established that infinitesimal material surface elements in a turbulent flow are preferentially stretched rather than compressed [9] and the mean rate of stretch of infinitesimal material surface elements in homogeneous isotropic turbulence scales with the Kolmogorov time scale, independently of the Reynolds number [10]. This picture is consistent with the dominant role of tangential strain, which is controlled by the rate of strain tensor and due to the preferential alignment of the surface normal with the eigenvector associated with the most compressive eigenvalue of the rate of strain tensor $[8,9,11]$.

In practical combustion applications, however, there are several complicating factors. Density and transport properties vary across the flame surface. In addition, the isoscalar surface associated with a reactive and/or diffusive scalar is neither infinitesimal nor material, since the surface propagates relative to the local velocity of the fluid. The most conspicuous outcome of propagation is the contribution of curved propagating surfaces to surface stretch. This mechanism is important to the destruction of flame surface [12-15]. Compared to the case of material (non-propagative) surfaces, the scaling of the contribution to flame stretch due to curvature is not at all obvious. Evidence in support of the statistics of surface stretch being independent of Reynolds number in turbulent flames is put forth by recent DNS studies of one-dimensional turbulent premixed flames [16].

To the authors' knowledge, the results presented are the first to examine numerically the statistics of flame surface stretch in a set of spatially evolving turbulent jet premixed flames with increasing Reynolds number at constant Karlovitz number. Realistic turbulent slot jet flows driven by shear are considered [3-5], rather than statistically one-dimensional flows with decaying [16] or forced turbulence [17-19] or low-shear Bunsen flames [20]. The set includes the simulation of a spatially evolving turbulent jet flame with the highest jet Reynolds number and scale separation achieved to date.

Section 2 presents a few mathematical preliminaries and Section 3 discusses the flame configuration and methods. Results are included in Section 4 and conclusions are given in Section 5.

\section{Mathematical preliminaries and definitions}

Firstly, we define a progress variable based on the mass fraction of molecular oxygen as $C=1-\left(Y_{\mathrm{O}_{2}}-\right.$ $\left.Y_{\mathrm{O}_{2}}^{b}\right) /\left(Y_{\mathrm{O}_{2}}^{u}-Y_{\mathrm{O}_{2}}^{b}\right)$, where $Y^{u}$ and $Y^{b}$ are the mass fractions of $\mathrm{O}_{2}$ in the unburnt and burnt mixtures, respectively. Next, we consider the constant property (isolevel) surface $C(\mathbf{x}, t)=c$ and define its Surface Density Function $(\mathrm{SDF})[1,21]$ as $\Sigma(c ; \mathbf{x}, t)=\langle|\nabla C| \delta[C-c]\rangle=$ $\langle|\nabla C| \mid C=c\rangle \mathcal{P}_{C}(c)$, where we rely on the fine-grained density function formalism [7]. Here, $\langle|\nabla C| \mid C=c\rangle$ is the conditional average of the magnitude of the gradient of the progress variable and $\mathcal{P}_{C}(c ; \mathbf{x}, t) d c$ is the probability of $C(\mathbf{x}, t)$ falling in the interval $[c, c+d c]$.

The SDF represents the average surface to volume ratio of the constant property surface $C(\mathbf{x}, t)=c[21]$. For a steady flow, its evolution equation reads [7]

$$
\nabla \cdot\left(\langle\mathbf{u}+S \mathbf{n}\rangle_{s} \Sigma\right)=\langle a+S \nabla \cdot \mathbf{n}\rangle_{s} \Sigma=\langle\mathrm{K}\rangle_{s} \Sigma,
$$

where $\mathbf{u}$ is the fluid velocity, $S$ is the displacement speed of the constant property surface, $\mathbf{n}=-\nabla C /|\nabla C|$ is the normal to the surface pointing in the direction of decreasing values of $C$, i.e. into the reactants, and $\mathrm{K}$ is the stretch rate of the constant property surface [7]. $\langle Q\rangle_{s}=\langle Q|\nabla C| \mid C=c\rangle /\langle|\nabla C| \mid C=c\rangle$ represents surface weighted conditional statistics.

The stretch rate term $\langle\mathrm{K}\rangle_{s} \Sigma$ acts as a source term, representing the growth (or destruction) rate of the area of the constant property surface. The stretch rate $\mathrm{K}$ consists of two components: $a=\nabla \cdot \mathbf{u}-\mathbf{n} \nabla \mathbf{u n}$ is the tangential strain rate and $S \nabla \cdot \mathbf{n}$ is commonly referred to as the 
curvature term, since $-\nabla \cdot \mathbf{n} / 2$ is the mean of the two principal curvatures of the two-dimensional constant property surface with normal $\mathbf{n}$ defined as above. The tangential strain rate $a$ represents the kinematic contribution of velocity to stretch, independently of surface propagation. The curvature term reflects the contribution of surface propagation in the presence of curvature.

The displacement speed takes the form $[13,14]$ :

$$
\begin{aligned}
S & =\frac{1}{|\nabla C|} \frac{D C}{D t}=\frac{\omega}{\rho|\nabla C|}-\frac{1}{\rho|\nabla C|} \nabla \cdot(\rho \mathbf{V C}) \\
& \approx \frac{\omega}{\rho|\nabla C|}+\frac{1}{\rho|\nabla C|} \frac{\partial}{\partial n}\left(\rho \mathcal{D} \frac{\partial}{\partial n} C\right)-\mathcal{D} \nabla \cdot \mathbf{n},
\end{aligned}
$$

where $\rho$ is the density, and $\mathbf{V}, \omega$ and $\mathcal{D}$ are the diffusion velocity, reaction rate and diffusion coefficient of the progress variable, respectively. In Eq. (3), the diffusive term in Eq. (2) has been decomposed into the normal diffusion and curvature diffusion terms [22] upon approximating the diffusion velocity with a closure of the form $\mathbf{V}=-\mathcal{D} \nabla C / C$. The curvature diffusion component emphasizes that curvature itself contributes linearly to the displacement speed.

\section{Flame configuration and numerical methods}

The flame configuration considered is a slot turbulent premixed jet flame surrounded by a coflow of burnt gases. The jet consists of a methane/air mixture with equivalence ratio $\Phi=0.7$ and temperature $800 \mathrm{~K}$. The background pressure is $4 \mathrm{~atm}$. The temperature and equivalence ratio have been selected to be close to the typical conditions at the exit of the compressor in gas turbines. An elevated value of the pressure is chosen to optimize the numerical configuration as it allows high Reynolds and Karlovitz numbers avoiding excessive inlet velocities and slot widths. Based on one-dimensional simulations of a freely propagating flame, the laminar flame speed is $S_{L}=1 \mathrm{~m} \mathrm{~s}^{-1}$ and the thermal thickness is $\delta_{L}=110 \mu \mathrm{m}$. The resulting flame time scale is $\tau_{L}=\delta_{L} / S_{L} \approx 110 \mu \mathrm{s}$.

The bulk velocity of the jet is $U=100 \mathrm{~m} \mathrm{~s}^{-1}$ and the coflow has a uniform velocity of $15 \mathrm{~m} \mathrm{~s}^{-1}$. Depending on case, the Reynolds number $\operatorname{Re}=U H / v$ varies from 2800 to 22400 as the slot's width $H$ increases from 0.6 to $4.8 \mathrm{~mm}$ ( $v$ is the kinematic viscosity of the reactants).

The flame configurations were selected in order to vary the Reynolds number, while keeping the Karlovitz number $\mathrm{Ka}=\delta_{L}^{2} / \eta^{2}$ approximately constant. The Kolmogorov scale is defined as $\eta=\left(\bar{v}^{3} / \tilde{\epsilon}\right)^{1 / 4}$, where $\tilde{\epsilon}$ is the Favre averaged energy dissipation and $\bar{v}=\overline{\mu / \rho}$ is the averaged viscosity. Because the turbulence intensity is a

\begin{tabular}{lcccc}
\hline & $\mathrm{R} 1$ & $\mathrm{R} 2$ & $\mathrm{R} 3$ & $\mathrm{R} 4$ \\
\hline $\mathrm{H}(\mathrm{mm})$ & 0.6 & 1.2 & 2.4 & 4.8 \\
$\mathrm{U}(\mathrm{m} / \mathrm{s})$ & 100 & 100 & 100 & 100 \\
$\delta_{\omega, 0}(\mu \mathrm{m})$ & 67.3 & 65.8 & 76.8 & 85.4 \\
$\mathrm{Re}$ & 2800 & 5600 & 11200 & 22400 \\
\hline$u^{\prime}(\mathrm{m} / \mathrm{s})$ & 14.3 & 10.1 & 9.9 & 11.7 \\
$l(\mathrm{~mm})$ & 0.54 & 0.54 & 0.67 & 1.1 \\
$\eta(\mu \mathrm{m})$ & 18 & 23 & 25 & 25 \\
$\tau_{\eta}(\mu \mathrm{s})$ & 4.0 & 6.5 & 7.3 & 7.3 \\
\hline$u^{\prime} / S_{L}$ & 14.2 & 10.0 & 9.8 & 11.6 \\
$l / \delta_{L}$ & 4.8 & 4.8 & 5.9 & 9.5 \\
$\mathrm{Ka}$ & 39 & 23 & 21 & 21 \\
$\mathrm{Re}$ & 49 & 39 & 40 & 50 \\
$\mathrm{Da}$ & 0.18 & 0.24 & 0.3 & 0.41 \\
\hline$N_{x}$ & 720 & 1440 & 2880 & 5760 \\
$N_{y}$ & 480 & 960 & 1920 & 3840 \\
$N_{z}$ & 256 & 256 & 512 & 1024 \\
\hline
\end{tabular}

Table 1: Simulations parameters. All scales of turbulence and related nondimensional groups are evaluated at the crosswise location where $\langle C\rangle=0.73$ and the streamwise location where detailed statistics of stretch and its components are presented $(x / L=0.6)$.

fraction of the bulk velocity with only a minor dependence on the Reynolds number, this scaling approach results in approximately a constant value of $u^{\prime} / S_{L}$ with increasing $l / \delta_{L}$ as the integral scale $l$ increases with the size of the jet width $H$. The streamwise evolution of the normalized turbulence intensity and integral scale are shown in a Borghi-Peters diagram included in the supplementary material.

A summary of all relevant flow parameters is provided in Tab. 1, where it is apparent that all flames lie in the thin reaction zone regime. Note that the turbulence properties for flame R1 deviate from the overall trend, possibly on the account of the very low Reynolds number of the configuration.

The size of the computational domain is $24 H$ in the streamwise $(x), 16 H$ in the crosswise $(y)$, and $4.3 H$ in the spanwise $(z)$ direction $(8.5 H$ for R1). The jet and the coflow streams are separated at the inlet by walls of thickness $H / 10$. The domain is periodic in $z$, open boundary conditions are prescribed at the outlet in $x$ and no-slip conditions are imposed at the boundaries in $y$. The inlet conditions for the velocity field are obtained from four auxiliary simulations of fully developed turbulent channel flow. The vorticity thickness at the exit $\left(\delta_{\omega, 0}\right)$ is reported in Tab. 1 .

The mesh is uniform in all three directions with a resolution $\Delta=20 \mu \mathrm{m}$, resulting in $88 \mathrm{M}(\mathrm{R} 1), 350 \mathrm{M}(\mathrm{R} 2)$, $2.8 \mathrm{~B}(\mathrm{R} 3)$, and $22 \mathrm{~B}(\mathrm{R} 4)$ grid points. The resolution is such that $\delta_{L} / \Delta \sim 6$ and $\Delta / \eta<2$ at all times. Cases R1 and R2 were simulated with double the spatial resolution also $(\Delta=10 \mu \mathrm{m})$ without any significant change in the statistics. An analysis of the resolution requirements 




Figure 1: Instantaneous snapshots of the mass fraction $\left(10^{2} \mathrm{ppm}\right)$ of the $\mathrm{O}$ radical (top row). Surface density function $\Sigma\left(\mathrm{mm}^{-1}\right)$ contour plot and mean progress variable field $\langle C\rangle$ isolines (bottom row) for all four flame configurations. The isocontours for $\langle C\rangle$ are $0.1,0.5$, 0.73 (cyan), and 0.8 .

is available in the supplemetary material.

The reactive, unsteady Navier-Stokes equations are solved in the low Mach number limit [23] with an established finite-difference solver suitable for use on massively parallel high-performance computing platforms [24]. The mixture obeys the ideal gas equation of state and all transport properties are computed with a mixture-average approach [25]. The simulations feature finite rate chemistry, described by a skeletal methane mechanism with 16 species and 72 reactions [26], and customized source code for the evaluation of the analytical Jacobian of the reactive source terms [27].

All simulations were performed on the Cray XC40 supercomputer "Shaheen" at King Abdullah University of Science and Technology, requiring about $100 \mathrm{M} \mathrm{cpu-}$ hours and executing on up to 131,072 processors. The database available for the analysis of the statistics exceeds 230 TB in size.

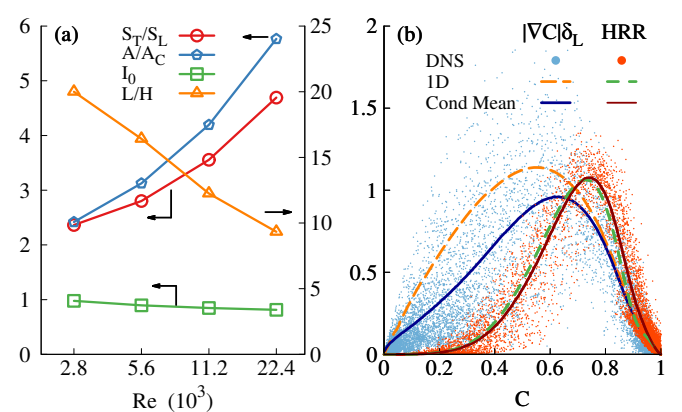

Figure 2: (a) Turbulent flame speed $S_{T} / S_{L}$, mean flame area $A / A_{c}$, correction factor $I_{0}$, and flame height $L / H$. (b) Scatter plot and conditional statistics of $|\nabla C|$ and heat release rate (HRR) in flame R3 and a one-dimensional laminar flame.

\section{Results}

\subsection{Overview of the flames}

The four turbulent premixed jet flames are shown in Fig. 1, where the SDF is reported alongside with selected isocontours of the mean progress variable $\langle C\rangle$. The SDF $\Sigma$ is defined for the constant property surface $C(\mathbf{x}, t)=0.73$, which is equal to the value of the progress variable corresponding to the peak heat release rate in a freely-propagating laminar flame.

In the remainder of the paper, this constant property surface will be referred to as flame sheet, although we acknowledge that the diffusive and reactive layers of a flame have finite thickness. Furthermore, considering that the flames fall in the thin reaction zone regime, the dependence of the surface statistics on the property value $c$ is addressed where appropriate.

Consistently with the recent DNS study of Wang et al. [28], $\Sigma$ is highest near the nozzle's exit plane and in the middle of the shear layer between the jet and the coflow. The flame brush is very narrow near the nozzle and widens rapidly moving downstream in the streamwise direction. As the flame brush widens, the surface density function $\Sigma$ decreases. The flame brush is widest on the centerplane at the tip of the flame. The spatial distribution of $\Sigma$ is qualitatively identical across flames, although its values decrease slightly from R1 to R4. In all four flames, the near field is best described as two spatially developing mixing layers up until $x / H \approx 6$, when $\langle C\rangle$ on the centerplane increases above zero.

The instantaneous snapshots of the mass fraction of the $\mathrm{O}$ radical in Fig. 1 display a clear trend of increasing separation of scales with Reynolds number. As far as the mean fields are concerned, the most obvious effect brought by the increase in Reynolds number from $\mathrm{R} 1$ to $\mathrm{R} 4$ is the decrease in the normalized flame height $L / H$, defined here as the distance between the exit plane 

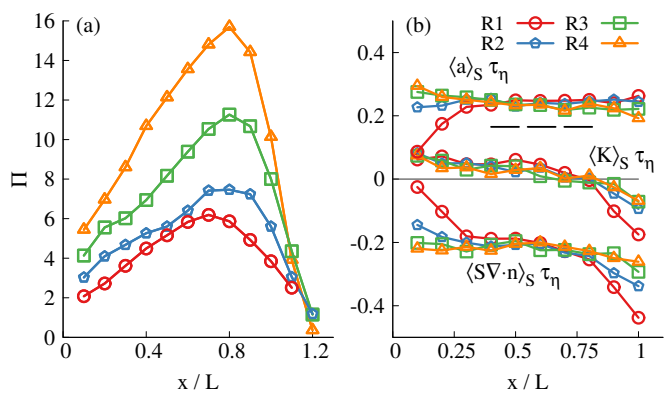

Figure 3: (a) Integral of $\Sigma$ in the crosswise direction, П. (b) surface averaged mean values of stretch, tangential strain rate and curvature term.

of the nozzle and the axial location where $\langle C\rangle=0.73$ on the centerplane. Notwithstanding changes in the flame height, flames R2, R3, and R4 display very similar mean envelopes, while flame R1 looks distinctly different, possibly on the account of the very low Reynolds number and a delay in transition.

A quantitative comparison of the burning rates across flames may be carried out based on the concept of turbulent flame speed for premixed jet flames. Let $\Omega$ be the volume integrated mass rate of consumption of methane, $A$ indicates the ensemble average of the area of the turbulent flame sheet, $A_{C}$ is the area mean surface $\langle C\rangle=0.73$, and $\rho_{f}$ is the density of methane in the reactants. Since the flames considered are envelope flames [2], we write $\Omega=\rho_{f} U W H$, where $W$ is the extent in the periodic spanwise direction. A normalized global turbulent flame speed is defined as $S_{T} / S_{L}=\Omega /\left(\rho_{f} S_{L} A_{C}\right)=\left(A / A_{C}\right) \Omega /\left(\rho_{f} S_{L} A\right)=\alpha I_{0}$, where $\alpha=A / A_{C}$ and $I_{0}=\Omega /\left(\rho_{f} S_{L} A\right)$ are the area ratio and the correction factor [2], respectively.

Figure 2(a) reports the values of $S_{T} / S_{L}, \alpha, I_{0}$, and $L / H$ for the four flames. Consistently with a decrease in the flame length $L / H$, which indicates a reduction in $A_{C} / H$, the turbulent flame speed $S_{T} / S_{L}$ increases. The area ratio $\alpha=A / A_{C}$ is also shown to increase with $\mathrm{Re}$, while $I_{0} \approx 1$ across flames. The observed increase of the turbulent flame speed with the Reynolds number, which in this case is related to an increase of the turbulent integral scale at constant $u^{\prime}$, is consistent with the theoretical analysis of Peters [29]. In addition, a levelling off of $S_{T} / S_{L}$, which would be expected at large Reynolds (large integral scale), is not observed in the present set of flames. Also this observation is consistent with the analysis of Peters [29], who shows that a significant flattening of the Reynolds dependency of $S_{T} / S_{L}$ should happen at about $l=100 / \delta_{L}$, which is one order of magnitude larger than the ratio achieved here.
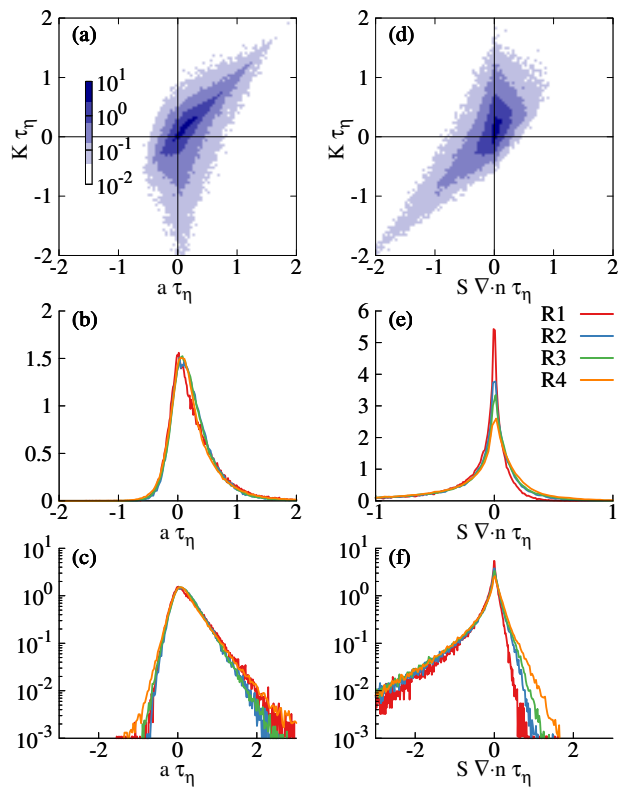

Figure 4: JPDF of stretch $\mathrm{K}$ and tangential strain rate $a=\nabla \cdot \mathbf{u}-\mathbf{n} \nabla \mathbf{u n}$ (a) and stretch $\mathrm{K}$ and curvature term $S \nabla \cdot \mathbf{n}$ (d) at $x / L=0.6$ for flame R4. The marginals of the tangential strain $(b, c)$ and curvature term $(\mathrm{e}, \mathrm{f})$ are shown for all four flames. All quantities are weighted by $|\nabla C| /\langle|\nabla C|\rangle_{s}$ and normalized by $\tau_{\eta}$.

As $S_{T} / S_{L}=I_{0} \alpha$ and $I_{0} \approx 1$, we conclude that the increase in the burning rate is due to the enhancement of the area of the flame sheet, rather than to modifications to the local reaction rates brought by turbulence. This interpretation is supported by the conditional mean of $|\nabla C|$ and heat release rate shown in Fig. 2(b) alongside with data from a freely propagating laminar flame. The conditional mean of the heat release rate is identical to that in the laminar flame and the $(C, \mathrm{HRR})$ samples cluster around the conditional mean, indicating that the turbulent premixed flames belong to the thin reaction zone regime. Notice that the progress variable value $c=0.73$ marks the location of peak heat release in both the laminar and the turbulent flames.

\subsection{Area and stretch of the flame surface}

We consider the evolution of the SDF and the scaling of the area of the flame sheet across configurations. We commence by defining the ensemble average of the area of the turbulent flame surface in the interval $[0, x]$ per unit extent in the spanwise direction $\widetilde{A}(x)$. This quantity is related to the volumetric integral of $\Sigma(x, y)$ as

$$
\widetilde{A}(x)=\int_{0}^{x} d x^{\prime} \int_{0}^{\infty} \Sigma d y^{\prime}=\int_{0}^{x} \Pi\left(x^{\prime}\right) d x^{\prime} .
$$




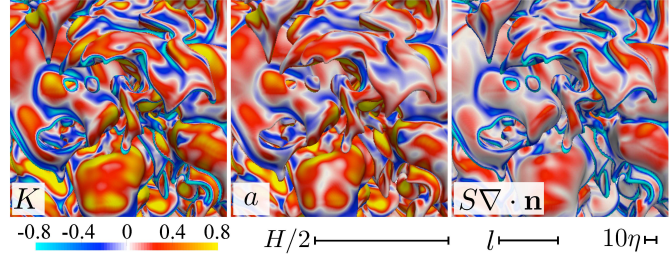

Figure 5: Instantaneous stretch rate $(K)$, tangential strain rate $(a)$, and curvature term $(S \nabla \cdot \mathbf{n})$ on the flame sheet at $x / L=0.6$ (flame R4). All quantities are weighted by $|\nabla C| /\langle|\nabla C|\rangle_{s}$ and normalized by $\tau_{\eta}$.

The variable $\Pi(x)=d \widetilde{A} / d x$ is shown in Fig. 3(a) versus the normalized streamwise distance $x / L$. Because $\Pi(x)$ is the integral of $\Sigma$ in the crosswise direction at all streamwise locations, it provides a concise manner of presenting the evolution of the flame surface area in the streamwise direction [14]. The streamwise evolution of $\Pi$ is qualitatively similar for all flames. It increases first, reaches a peak value at $x / L \approx 0.8$, and then decreases towards zero. From R1 to R4, the crosswise integral of the SDF increases at all streamwise locations due to the larger physical size of the flame brush associated with the larger slot width.

An explanation for the streamwise evolution of $\Pi$ and associated $\Sigma$ is provided by the statistics of stretch $K$ and its components shown in Fig. 3(b). All statistics are averaged in the crosswise direction and weighted by $|\nabla C|$, thereby providing surface averages. Normalization is by the local value of the Kolmogorov time scale $\tau_{\eta}$. The Kolmogorov time is computed as $\tau_{\eta}=(\bar{v} / \tilde{\epsilon})^{1 / 2}$. For each streamwise location, the value of $\tau_{\eta}$ used for the normalization is selected at the position where the mean progress variable is $\langle C\rangle=0.73$. As shown, the surface averaged mean tangential strain rate $\langle a\rangle_{s}$ is approximately constant in the streamwise direction and positive, indicating production of surface in the mean. In contrast, the surface averaged mean curvature term $\langle S \nabla \cdot \mathbf{n}\rangle_{S}$ is negative at all locations, indicating that it is responsible for destroying the flame surface.

The positive value of $\langle a\rangle_{s}$ is consistent with the wellknown preference for stretching over compression of surfaces in turbulent flows. Based on the data from our simulations, we find $\langle a\rangle_{s} \tau_{\eta} \approx 0.23$, which is in close agreement with the mean tangential rate of strain $\langle a\rangle_{s} \tau_{\eta}=0.165$ reported by Girimaji and Pope [10] for infinitesimal material surfaces in homogeneous isotropic turbulence ( $\left.38 \leq \operatorname{Re}_{\lambda} \leq 93\right)$. The differences between the two values may be due to various factors, including mean shear, variable density, and the propagative nature of the flame surface. In particular, the positive mean velocity divergence may be responsible for the slightly larger value of $\langle a\rangle_{s} \tau_{\eta}$ in turbulent flames compared to isothermal turbulence. Nonetheless, our data suggest that the statistics of the tangential strain of the flame surface are controlled mostly by the kinematics of the turbulent velocity field, which induce a preferential alignment of the surface normal with the eigenvector associated with the most compressive eigenvalue of the rate of strain tensor $[8,9]$. This mechanism and associated statistics appear to be robust and largely applicable to isothermal and variable density flows alike.

The sum of $\langle a\rangle_{s}$ and $\langle S \nabla \cdot \mathbf{n}\rangle_{s}$ is the surface averaged stretch $\langle K\rangle_{s}$, which is positive up to $x / L \approx 0.8$ and negative thereafter. It is apparent that the two components of stretch are large in magnitude, although their sum is small. Thus, the sign of $\langle K\rangle_{s}$, which indicates whether the area of the flame surface grows or decreases on average, is governed by a rather small difference between the large contributions of surface production and surface destruction. Wang et al. [14] present very similar trends in a lean methane/air turbulent premixed jet flame issuing from a round nozzle at very high Karlovitz numbers. The similarity of our results and those at higher Karlovitz number suggests that these mechanisms are independent of $\mathrm{Ka}$ and possibly insensitive to the regime of turbulent premixed combustion.

As shown in Fig. 3, the statistics of stretch and its components are largely independent of the Reynolds number when normalized by $\tau_{\eta}$. In particular, the normalized stretch for the R3 and R4 flames collapse one on the other within statistical errors. This behavior is consistent with surface stretch being related to velocity gradients, which scale with the Kolmogorov scale $[8,10]$.

The statistics differ the most across flames in the near field, where the flow is possibly transitioning from a channel to shear-driven turbulence. This explanation is supported by the fact that the statistics for the flame with the lowest Reynolds number (R1) are distinctly different. Nonetheless, it is not clear at present which characteristic length is best suited to nondimensionalize the streamwise coordinate, so that there exists some ambiguity with regard to the interpretation of the spatial variation of the statistics across flames.

The joint probability density functions (JPDF) of the pairs $(K, a)$ and $(K, S \nabla \cdot \mathbf{n})$ and their marginal PDFs are shown in Fig. 4(a,d) for flame R4 at $x / L=0.6$. Statistics are complemented by Fig. 5, where the instantaneous flame sheet, colored by stretch rate, tangential strain rate, and curvature term, is shown for a small region centered at $x / L=0.6$.

At the $x / L=0.6$ location, the mean stretch $\langle K\rangle_{s}$ is positive. The JPDFs indicate unequivocally that intense 
positive stretch events (surface generation) are due to the tangential strain rate, while intense negative stretch events (surface destruction) are due to the curvature term. The tangential strain rate is also responsible for moderate stretch events. These conclusions are consistent with the behavior of the means in Fig. 3(b).

Figure 5 shows qualitatively that most of the flame sheet experiences moderate positive stretch, due to the tangential strain rate. Regions of large negative stretch are confined to very narrow ridges and locations where the surface has a large curvature and the flame sheet is cylindrical in shape $[30,31]$. Over appreciable portions of the flame sheet, the curvature term $S \nabla \cdot \mathbf{n}$ is also responsible for localized surface production, augmenting the contribution of the tangential strain.

The marginal PDF of the tangential strain has a positive mean and positive most probable value (Fig. 4(b,c)). The marginal PDF of the curvature term (Fig. 4(e,f)) shows that the most probable value is positive, although the mean is negative. As in the case of the mean stretch and its components, the conclusions above hold across all Reynolds numbers and the mean and most probable values of each of the marginal PDFs are nearly independent of the Reynolds number when normalized by $\tau_{\eta}$. Minor differences are apparent in the tails of the distributions, especially for the curvature term. As the Reynolds number increases, the tails of the PDFs widen (Fig. 4(c,f)), in particular for the positive values of the propagative term. Considering that the Damköhler number of the different cases increases with increasing Reynolds number, it is not possible to ascribe the widened tails to low Damköhler number effects. In view of the Kolmogorov scaling observed for the stretch componenents, it is reasonable to expect that intense events, which dominate the tails of the PDFs, follow the classical scaling of Kolmogorov turbulence, which is characterized by larger probability of intense events for increasing Reynolds number, consistently with small scale turbulence intermittency [9, 32].

As discussed, the surface averaged mean of the curvature term $\langle S \nabla \cdot \mathbf{n}\rangle_{s}$ is negative, indicating that this term dominates the process of surface destruction. Figure 6 shows the JPDF of the two factors contributing to the curvature term, $S$ and $\nabla \cdot \mathbf{n}$. The marginals of each variable are also shown for all flames.

A negative value of the curvature term, corresponding to destruction of surface, requires that $S$ and $\nabla \cdot \mathbf{n}$ have opposite signs, i.e. events that occur in the second (II) and fourth (IV) quadrants in Fig. 6. This occurs when the flame is advancing into the reactants $(S>0)$ and the center of curvature is on the side of the reactants or when the flame is receding from the reactants $(S<0)$



Figure 6: JPDF of $S$ and $\nabla \cdot \mathbf{n}$ for flame R4 at $x / L=0.6$. The most probable values (white solid circle) and the mean values (white cross) are marked. The marginals are shown for all flames.

and the center of curvature is on the side of the products $(\nabla \cdot \mathbf{n}>0)$. Other combinations in the first (I) and third (III) quadrants lead to surface production.

The data in Fig. 6 show that the most probable values of the variables $(S, \nabla \cdot \mathbf{n})$ fall in a region of the III quadrant near the vertical axis, i.e. $S<0$ and $\nabla \cdot \mathbf{n} \approx 0$. In this region, the contribution of the curvature diffusion term is negligible and the reaction and normal diffusion terms dominate (Eq. (3)). Since the former is always positive, we conclude that the occurrence of $S<0$ is due to events in which the negative normal diffusion term exceeds in magnitude the reaction term. In contrast with the most probable value, $\langle\rho S\rangle / \rho_{u} S_{L}$ is positive and $\approx 1$ as expected ( $\rho_{u}$ is the density of the reactants).

The marginal PDFs of $\rho S / \rho_{u} S_{L}$ are broadly distributed and do not show any prominent peak at 1 (Fig. 6). Furthermore, there are differences across flames, with data from the highest Reynolds number displaying broader tails. At the highest Reynolds number considered, the PDF shows two distinct maxima at positive and negative values of $S$. These observations suggest that, although $\langle\rho S\rangle / \rho_{u} S_{L} \approx 1$, the statistics of $S$ are affected strongly by turbulence in general.

From the data in Fig. 6 and the discussion above, it is apparent that the statistics of the two factors $S$ and $\nabla \cdot \mathbf{n}$ are nuanced. Figure 7(a) provides a detailed analysis of the contributions of each quadrant to the surface averaged mean $\langle S \nabla \cdot \mathbf{n}\rangle_{s}$ and shows that samples in the II quadrant contribute the most, followed by pairs in the IV quadrant $(S<0$ and $\nabla \cdot \mathbf{n}>0)$. The remaining two cases (events in the I and III quadrants) are responsible for surface production and contribute the least.

The probabilities of each event are shown in Fig. 7(b). Samples of displacement speed and curvature are most 

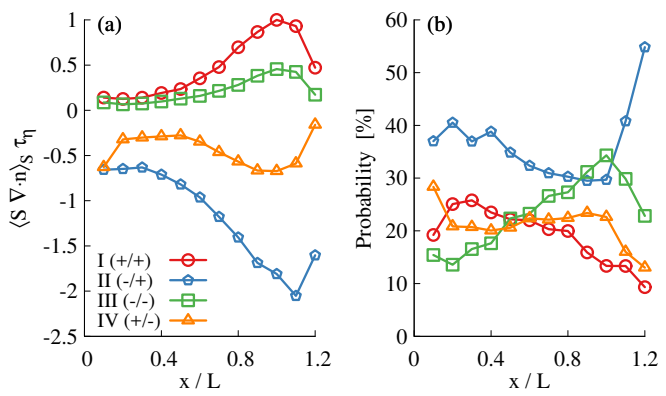

Figure 7: (a) Surface averaged mean contribution to $\langle S \nabla \cdot \mathbf{n}\rangle_{S}$ by samples in each quadrant. (b) Probability of events occurring in each quadrant.

likely to fall in the II quadrant, with probabilities exceeding $40 \%$ near and beyond the flame tip. Events in quadrant IV are very likely in the near field, but their probability decreases in the streamwise direction. The probability of events in the III quadrant, leading to flame production, is very low near the nozzle exit plane, increasing up to $35 \%$ in the streamwise direction. Yet, events in the III quadrant contribute little to production. The opposite is true for events in the I quadrant, which are less likely ( $\leq 25 \%$ and $\approx 10 \%$ at the flame tip) but contribute the most to surface production.

\section{Conclusions}

The data from four DNS of turbulent premixed slot jet flames up to $\mathrm{Re}=22.4 \times 10^{3}$ show that tangential strain is responsible for surface production and flame propagation in the presence of curvature contributes to surface destruction. The mean rates of stretch associated with the two mechanisms are shown to scale with the Kolmogorov time scale, independently of the Reynolds number and location in the jet. This picture is consistent both qualitatively and quantitatively with the dynamics and stretch statistics of infinitesimal material (nonpropagative) surfaces in homogeneous isotropic turbulence. The flame surface is found to be mostly flat and undergoing positive stretch, while surface destruction is due to reactive fronts burning into the reactants at tight cylindrical folds. The most probable value of the displacement speed, which is positive in the mean as expected, is shown to be negative, consistently with turbulence stretching the flame surface, steepening scalar gradients, and enhancing mixing. Finally, the PDF of the displacement speed broadens as the Reynolds number increases.

\section{References}

[1] D. Veynante, L. Vervisch, Prog. Energ. Combust. Sci. 28 (2002) 193-266.

[2] J. F. Driscoll, Prog. Energ. Combust. Sci. 34 (2008) 91-134.

[3] R. Sankaran, E. R. Hawkes, J. H. Chen, T. Lu, C. K. Law, Proc. Combust. Inst. 31 (2007) 1291-1298.

[4] E. R. Hawkes, O. Chatakonda, H. Kolla, A. R. Kerstein, J. H. Chen, Combust. Flame 159 (2012) 2690-2703.

[5] P. Trisjono, H. Pitsch, Proc. Combust. Inst. 36 (2017) 20332043.

[6] S. Luca, A. Attili, F. Bisetti, 55 th AIAA Aerospace Sciences Meeting, (AIAA 2017-0603) (2017) 0603.

[7] L. Vervisch, E. Bidaux, K. Bray, W. Kollmann, Phys. Fluids 7 (1995) 2496-2503.

[8] A. Tsinober, An Informal Conceptual Introduction to Turbulence, Springer, Netherlands, 2009.

[9] A. S. Monin, A. M. Yaglom, Statistical Fluid Mechanics, Volume II: Mechanics of Turbulence, Dover Publications, Mineola, NY, 2007.

[10] S. S. Girimaji, S. B. Pope, J. Fluid Mech. 220 (1990) 427-458.

[11] W. T. Ashurst, A. R. Kerstein, R. M. Kerr, C. H. Gibson, Phys. Fluids 30 (1987) 2343-2353.

[12] A. Trouvé, T. Poinsot, J. Fluid Mech. 278 (1994) 1-31.

[13] N. Chakraborty, R. Cant, Phys. Fluids 17 (2005) 065108.

[14] H. Wang, E. R. Hawkes, J. H. Chen, B. Zhou, Z. Li, M. Aldén, J. Fluid Mech. (2017) 511-536.

[15] G. Nivarti, S. Cant, Proc. Combust. Inst. (2017) 1903-1910.

[16] N. Chakraborty, R. Cant, Proc. Combust. Inst. 34 (2013) 13471356.

[17] S. Lapointe, G. Blanquart, Combust. Flame (2016) 294-307.

[18] A. Y. Poludnenko, E. S. Oran, Combust. Flame (2011) 301-326.

[19] P. E. Hamlington, R. Darragh, C. A. Briner, C. A. Z. Towery, B. D. Taylor, A. Y. Poludnenko, Combust. Flame (2017) 193207.

[20] J. B. Bell, M. S. Day, J. F. Grcar, M. J. Lijewski, J. F. Driscoll, S. A. Filatyev, Proc. Combust. Inst. 31 (2007) 1299-1307.

[21] S. Pope, Int. J. Engng Sci. 26 (1988) 445-469.

[22] T. Echekki, J. H. Chen, Combust. Flame 118 (1999) 308-311.

[23] A. Tomboulides, J. Lee, S. Orszag, J. Sci. Comput. 12 (1997) 139-167.

[24] O. Desjardins, G. Blanquart, G. Balarac, H. Pitsch, J. Comput. Phys. 227 (2008) 7125-7159.

[25] A. Attili, F. Bisetti, M. E. Mueller, H. Pitsch, Combust. Flame 166 (2016) 192-202.

[26] S. Luca, A. N. Al-Khateeb, A. Attili, F. Bisetti, J. Propul. Power (2017) 1-8.

[27] F. Bisetti, Combust. Theor. Model. 16 (2012) 387-418.

[28] H. Wang, E. R. Hawkes, B. Zhou, J. H. Chen, Z. Li, M. Aldén, Proc. Combust. Inst. 36 (2017) 2045-2053.

[29] N. Peters, J. Fluid Mech. 384 (1999) 107-132.

[30] L. Cifuentes, C. Dopazo, J. Martin, C. Jimenez, Phys. Fluids (2014) 065108.

[31] R. A. C. Griffiths, J. H. Chen, H. Kolla, R. S. Cant, W. Kollmann, Proc. Combust. Inst. 35 (2015) 1341-1348.

[32] K. R. Sreenivasan, R. A. Antonia, Annu. Rev. Fluid Mech. 29 (1997) 435-472. 